\title{
An Efficient Image Denoising Technique for Unprocessed Raw Images using Combine Linear and Non-Linear Filtering
}

\author{
Anichur Rahman \\ Lecturer \\ Department of Computer Science and Engineering \\ National Institute of Textile Engineering and Research \\ Savar, Bangladesh \\ Md. Razaul Karim \\ Department of Computer Science and Engineering \\ Mawlana Bhashani Science and Technology University \\ Tangail, Bangladesh
}

\author{
Md. Anwar Hussen Wadud \\ Lecturer \\ Department of Computer Science and Engineering \\ Bangladesh University of Business and Technology \\ Dhaka, Bangladesh \\ Md. Wahidur Rahman \\ Department of Computer Science and Engineering \\ Mawlana Bhashani Science and Technology University \\ Tangail, Bangladesh
}

\author{
Mohd. Sultan Ahammad \\ Lecturer \\ Department of Computer Science and Engineering \\ Mawlana Bhashani Science and Technology University \\ Tangail, Bangladesh
}

\begin{abstract}
Image denoising is used to improve the accuracy and quality of an image. Removing noise from the original image is still challenging for researchers. In this research, an efficient algorithm capable of removing noise from "unprocessed" or raw images is proposed. The algorithm supplants the noise by the median of averages found from a special combination of the pixels. After that, to evaluate the performance of image authors has calculated the Signal to Noise Ratio (SNR), the Mean Square Error(MSE), Root Mean Square Error (RMSE), the Root Mean Square Signal to Noise Ratio (RMS_SNR), Image Fidelity (IFY). Finally, the proposed filtering technique gives a better result with comparison to other existing filtering techniques (Median, Average, Mean, etc).
\end{abstract}

\section{General Terms}

Denoising, Linear Filtering, Raw Images et. al.

\section{Keywords}

Image Processing, Image Denoising, Raw Image, Filters, SNR, MSE, RMSE, Performance Evaluation etc.

\section{INTRODUCTION}

Images are authoritative and the exercise of using digital images has been changed. As the imaging devices are not good enough, they produce different impairments. Noise is the most common im- pairment. It is very crucial to denoise the images. There are piles of algorithms works with different types of noise models [9].

Ultrasound Imaging is used to alleviate quick diagnosis and the images contain dozens of noise. Existing denoising techniques have some limitations [21]. Ultrasound images are damaged by speckle noise [15]. Most of the imaging system of the liver and kidney because of the small structure of organs. Sometimes the edge of an image needs to be detected. Especially in a medical image, edges are very important to define the shape of an object and edge detection is somehow dependent on the noise level. In real-time communication multispectral image plays a critical role which normally contains noises of a different model. This phenomenon disturbs the processing of MSIs [12]. That's why denoise the images that are needed. Images can be corrupted by impulsive noise and furthermore, the noisy image can't be used for the diagnostic purpose which is a very large area of image processing.

There exists a bunch of techniques to denoise images. As digital image processing deals with the pixel values, it is not so easy to find out the differences between the noise and the actual properties of the image. Maximum denoising techniques [14] remove the tiny details from the image. Though some proficiency move out the noise smoothly, it is still a matter of thinking about the noise reduction process.

Research is running to upgrade the performance of the denoising algorithm [16], [17], [25], [26]. As mentioned before, researchers tried to get the best proficiency among the filtering techniques with various classes of images. Aerial images are likely to suffer from Gaussian, salt and pepper noise, etc. Peak Signal to Noise Ratio 
(PSNR) and Mean Square Error (MSE) is used to compare the quality of the living filtering technique [23].

Linear filtering [7] techniques result in blur images. It could remove the edges with fine detail from the images. Blur image can not be the desired one anyway. Tons of people work on different processes and a different algorithm and got a good result. Existing filters like mean, median, etc. remove the noise with some specific noise model. In the proposed Algorithm, authors apply both the mean and median with a novel combination. Here an algorithm is provided followed by a flow chart describing the whole process. The rule for the Raw image file has been applied. The SNR, MSE, RMS_SNR, RMSE, and IFY into account to realize the performance of the algorithm have been taken.

This paper has been organized as follows: Related works have been discussed in Section II. Section III provides the background which includes noise detection, noise removing techniques and evaluation criteria. Then denoising techniques including the proposed algorithm and flowchart have been proposed in Section IV. After that, the experiment and result analysis are presented in Section V. Finally, the paper has been concluded in Section VI with the significance and future implications.

\section{RELATED WORKS}

Several pieces of research have been proposed in recent years based on image processing especially in the field of image denoising. In paper [3] the authors proposed a machine learning approach on a single-image denoising algorithm. In order to reach this goal the authors presented a significant way to work on an unprocessed image using image processing pipelines in a response to inverting a single step of images. Moreover, the authors also depicted an effective model that would provide training to all the topical processing on the eve of evaluating loss function for denoising. The authors also assure experimented data analysis using the Convolution Neural Network (CNN) and find the lower error rates of 14-38 percent. The author of the paper [1] provides a short review of image denoising techniques. Advantages, as well as disadvantages of image denoising techniques, were also tried to figure out. The authors also tracked out different types of denoting techniques and compared them with several types of existing solutions. Finally, this paper inferred a which denoising method is very effective for the image denoising process. The authors of the paper [21] worked on a 3D image. The key idea of these works were to despeckle an ultrasound image. To meet these, the authors had used a linear regression model to eliminate speckle noise. The authors claimed that the proposed methodology far more efficient than existing solutions especially for physicians to diagnosis properly. Again, intellectual properties were generated through this proposed system. To track Speckle noise of an image, the authors of paper [5] worked with ultrasound images. The authors had used Fuzzy Logic-based Techniques (FLT) for speckle noise detection. The key idea of using the method was to replace upper pixel value into lower pixel value through the help of neighboring pixels. In order to get a better result, the authors had maintained a methodology called Binning Method which effectively reduces the noise of an image. This method result was calculated through SNR, MSE, PSNR and Edge Preservative Factor (EPF). Experimented data analysis showed improved performance than the existing methodology. In [22] the authors removed the noise using edge detection methodology. Edge detection was obtained through the use of different types of filters. Because of analyzing an image for edge detection SNR, PSNR, RMS, and Image Fidelity were calculated. These edge detection operators provided a much better result than existing edge detection operators.

The authors of paper [6] proposed an unprecedented mechanism to process an image using different types of filtering techniques through image restoration. The authors aimed at the digital image to rebuild an image in its regular form from the noisy image. The significance of this work was an overview based image restoration process. To summarize, Histogram Adaptive Fuzzy (HAF) was used and compared the tested data with several types of filtering processes such as Adaptive Fuzzy Mean Filter (AFMF), Weighted Fuzzy Mean (WFM) and Min-Max Exclusive Mean (MMEM) [19] [18]. The authors also interpreted the capabilities of corresponding methods and notified the results accordingly. In the paper, $[8]$ the authors had used two efficient algorithms called Centerto- Boundary (CB) and Bound-to-Boundary (BB). These methodologies had designed for $n \times n$ mask, though $3 \times 3$ mask was used for respective implementation certainly required for image convolution process. BB and CB algorithms were utilized and compared with traditional average filtering techniques [10] along with four parameters namely MSE, PSNR, EMF, and RSME. This proposed procedure was successfully tested in 1000 images and found a superior result. The proposed algorithms identically enhanced the quality of the images. The aim of paper [24] was to eliminate Gaussian noise from an image. The key purpose of this work was to build an effective solution to remove noise from an image with two dispute parameters namely edge preservation and computational complexity. The contribution of this proposed algorithm had been compared with several types of filtering techniques such as bilateral filter, Kmeans filter, wiener filter, alpha trimmed mean filter, and trilateral filter and found remarkably improved results on basic objective and subjective evolvement.

The authors of paper [11] had proposed a momentous non-linear image filtering technique to denoise an image. The working criteria of this proposed methodology were to change a corrupted pixel through the value of the its median or the value of processed neighboring pixels. This proposed methodology was also simulated and found the ability to eliminate the impulse noise of an image more than 70 percent. The authors also tested the respective methods on different types of images and listed corresponding obtained data. In paper [9] shows a survey on image denoising techniques. The authors tried to figure out how noise affects a high-quality image, why it has to use image denoising due to removing noise, what the traditional methods for image denoising and how to improve better image using denoising techniques.

\section{BACKGROUND}

Image denoising is a crucial phenomena when it is for the real time and raw medical images especially [13]. There are many noise filtering processes like mean filter, median filter [4], [20], etc. Most of the process results in blured and distorted features. From the above premises, an efficient denoising technique that will efficiently kick out the impulse noise from the raw image that have been proposed.

\subsection{Noise Detection Technique}

In this section, authors sense the image and construct a $3 \times 3$ test window containing noisy pixels. Several combinations of pixels are considered from this test window to compute the average values. Average values are used to filter the image. 


$$
\mathrm{X}_{\mathrm{T}}=\left[\begin{array}{lll}
\mathrm{X}_{1,1} & \mathrm{X}_{1,2} & \mathrm{X}_{1,3} \\
\mathrm{X}_{2,1} & \mathrm{X}_{2,2} & \mathrm{X}_{2,3} \\
\mathrm{X}_{3,1} & \mathrm{X}_{3,2} & \mathrm{X}_{3,3}
\end{array}\right]
$$

\subsection{Noise Removing Techniques}

Three (3) average values have been calculated from different patterns of previously selected pixels with corruption. The vertical, horizontal, diagonal or any other complexion that has been taken. For example:

$$
\begin{aligned}
& \mathbf{X}_{1,1}+\mathbf{X}_{1,2}+\mathbf{X}_{1,3} \\
& \mathbf{X}_{2,1}+\mathbf{X}_{2,2}+\mathbf{X}_{2,3} \\
& \mathbf{X}_{3,1}+\mathbf{X}_{3,2}+\mathbf{X}_{3,3}
\end{aligned}
$$

Then the averages have been calculated and sort them. The filtering has been finished by replacing the current pixel by the mid average value. Thus it works with the mean and median simultaneously.

$$
\begin{gathered}
\mathbf{A v g}=\left[\mathbf{X}_{\mathbf{i j}}+\mathbf{X}_{\mathbf{i}, \mathbf{j}+1}+\mathbf{X}_{\mathbf{i}, \mathbf{j}+1}\right] / \mathbf{3} \\
\mathbf{A v g}=\operatorname{Sort}(\mathbf{A v g})
\end{gathered}
$$

$$
\text { Midvalue }=\mathbf{A v g}[\mathbf{2}]
$$

\subsection{Evaluation Criteria}

The performance is conveyed by means of some statistical models. The SNR, MSE, RMSE, RMS_SNR, and IFY have been used. SNR is the signal to noise ratio defined by the equation which shown in below:

$$
S N R=\left[\frac{\sum_{x=1}^{M} \sum_{y=1}^{N} f_{2}(x, y)^{2}}{\sum_{x=1}^{M} \sum_{y=1}^{N}\left(f_{2}(x, y)-f_{1}(x, y)\right)^{2}}\right]
$$

Where $f_{1}(x, y)$ is the input image and $f_{2}(x, y)$ is the processed image. Each image contains $\mathrm{M}$ rows and $\mathrm{N}$ columns. Higher SNR causes better image.

MSE is used to compute RMSE which is another parameter to take decision whether an image is good enough or not.

$$
\begin{gathered}
M S E=\left[\frac{\sum_{\mathrm{x}=1}^{\mathrm{M}} \sum_{\mathrm{y}=1}^{\mathrm{N}}\left(\mathrm{f}_{1}(\mathrm{x}, \mathrm{y})-\mathrm{f}_{2}(\mathrm{x}, \mathrm{y})\right)^{2}}{M \cdot N}\right] \\
R M S E=\sqrt{M S E}
\end{gathered}
$$

RMS_SNR is calculated from the formula. It is the root mean square of signal to noise ratio.

$$
R M S_{-} S N R=\sqrt{\left[\frac{\left.\sum_{x=1}^{M} \sum_{y=1}^{N} f_{2}(x, y)\right)^{2}}{\sum_{x=1}^{M} \sum_{y=1}^{N}\left(f_{2}(x, y)-f_{1}(x, y)\right)^{2}}\right]}
$$

IFY is the Image fidelity that defines the image quality of faithful.

$$
I F Y=1-\frac{1}{S N R}
$$

\section{PROPOSED DENOISING TECHNIQUE}

\subsection{Proposed Algorithm}

The proposed algorithm takes a raw image and some statistical measurements to compare with the result of the proficiency. Comparing the numerical values, the algorithm returns the best measurements with the combination of the pixels used to compute the averages and the median of the averages which is used to replace the image pixel for processing. In the first place, it reads a raw image and starts to process the whole image over and over. In each iteration, a $3 \times 3$ window is defined as the filtering window and a data structure to store and sort the averages. One iteration goes through the pixels of an image and calculates 3 averages with a different combination of pixels.

For example, the pixels horizontally, vertically, or diagonally are chosen. One may choose any other combinations. Substituting the image pixels, a new processed image and then the numerical values are calculated (SNR, MSE, etc.). Finally, the measurements have been compared with the previously provided values and when the parameters are good enough, the algorithm returns the parameters and saves the combinations of pixels for later use. In algorithm, the best parameters have been gotten for the combinations given in Fig. 1

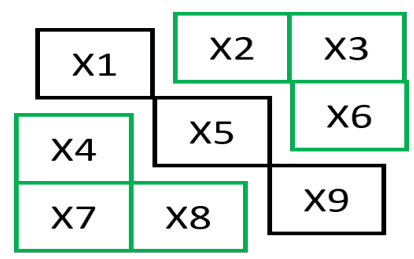

Fig. 1. Best Combination of Pixels

First of all, three average values say a1 (x2,x3,x6), a2 (x1,x5,x9) and $\mathrm{a} 3(\mathrm{x} 4, \mathrm{x} 7, \mathrm{x} 8)$ have been calculated. The pixel combination is given in the parentheses as in Fig. 1 After sorting the averages then the main image pixel with the mid-value among the averages has been replaced. Thus the best result has been gotten.

\subsection{Proposed Flowchart}

A flowchart has been constructed so that understanding the workflow of the algorithm and the entire process. The flowchart defines the process clearly with the directions of the arrows. The flowchart shows that it needs to choose a combination and assign some structure initially to control and perform the process.

The image is scanned until there remains any pixels. For every pixels in the image, averages are calculated and replaced the current pixel with the median of the averages. After finishing the scan, The statistical terms are measured and compared with the previous one. If the parameters satisfies the condition then the work flow is terminated and the values are returned as documentation. The proposed flowchart is given below in Fig. 2 


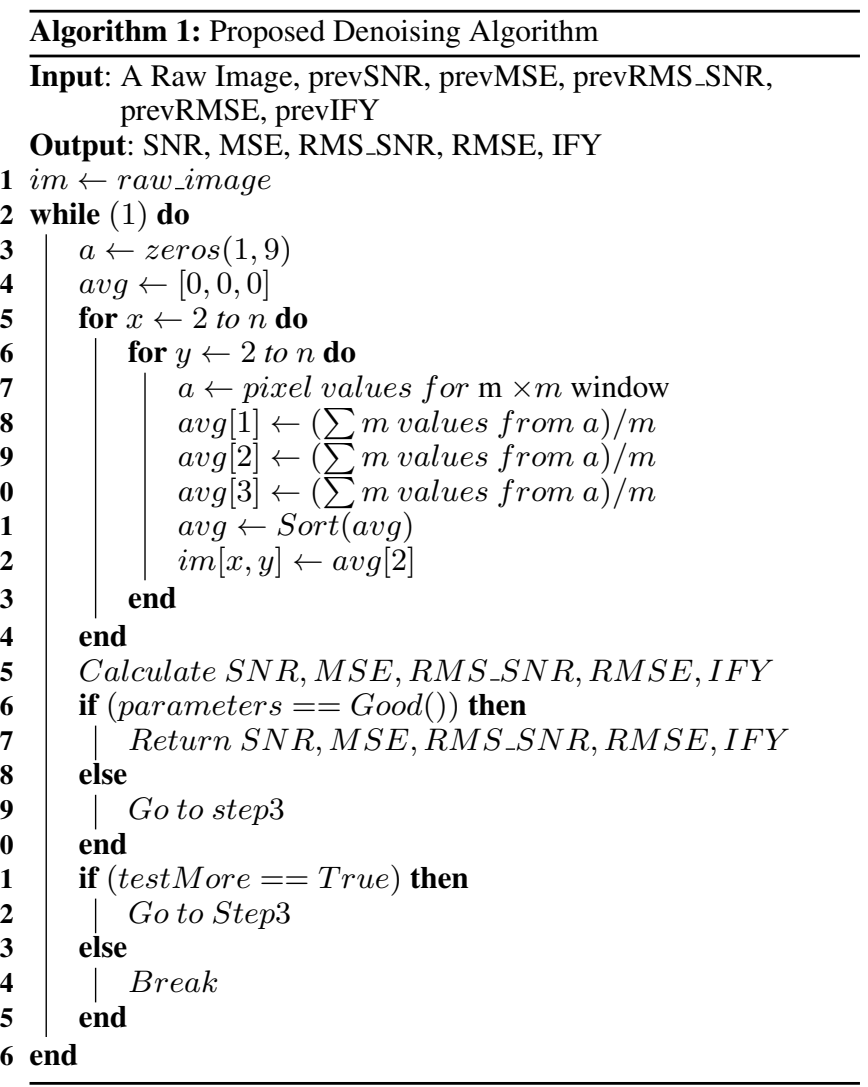

\section{EXPERIMENT AND RESULTS ANALYSIS}

\subsection{Visual Compare}

The algorithm on a raw image and visualized the subjective performance have been applied in Fig. 3 Here, the traditional noise removing filter is applied too. The figure shows the original raw image before filtering (a), result of the traditional filtering technique (b), and the result from proposed method (c). Another example is shown in the Fig. 4 Fig. 4 (4) shows the original raw image of carotid artery. After processing the image with existing filtering technique, the result shown in Fig. 4 (b). The result from proposed method, Fig. 4 c) seems more brighter than the previous one which is easy to visualise. Comparing the images from the figure and subjectively the result of this method is better. Moreover, the statistical comparison are considered for better understanding.

\subsection{Statistical/Numerical Compare}

To evaluate the efficiency of the Proposed Method, the simulation study has been approved using MATLAB [8]. One excellent brain noisy image (Raw Image) is selected for simulation learning [2]. The Propose method applies to the 2D raw image which provides a good result compared with the traditional filtering method. This proposed method is compared with the existing method which is shown in Table 1.

From the Table, the proposed filtering technique provides better results than the existing filtering techniques. Because for given image (noisy brain raw image) measurement criteria proposed filter provides better results than existing filters.

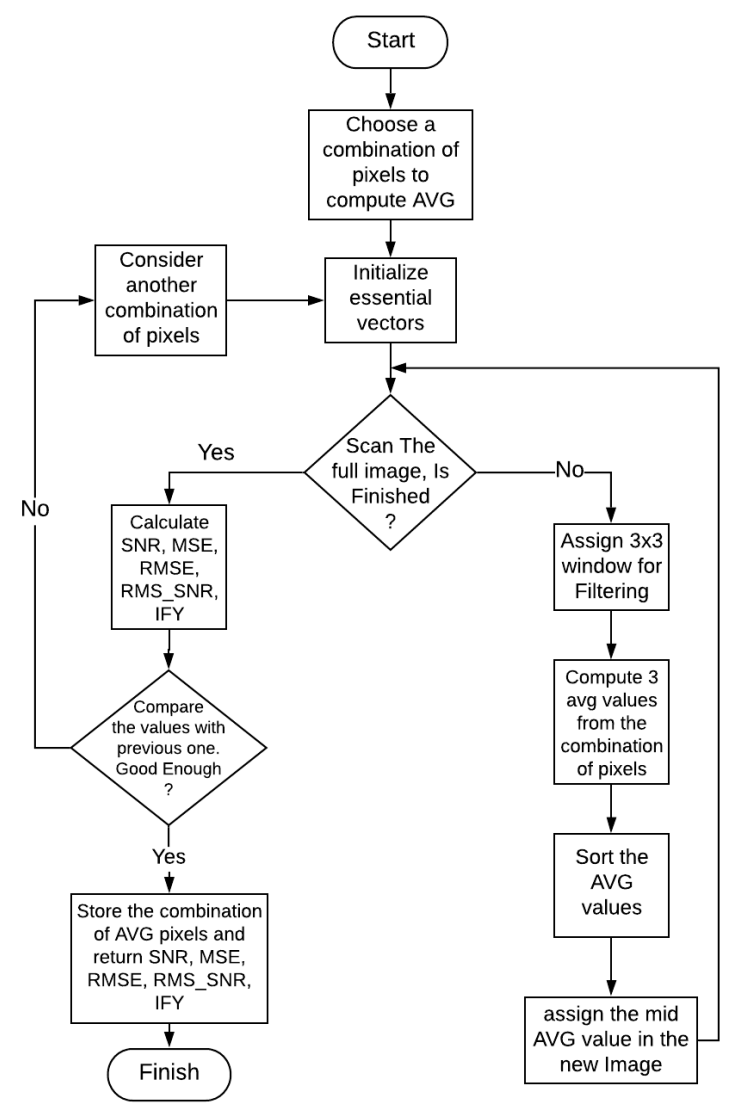

Fig. 2. Flowchart of the Proposed Method

Table 1. Comparison of Existing Methods and Proposed Method for Brain Noisy Images

\begin{tabular}{|c|c|c|c|c|}
\hline Values & Median & Average & Mean & $\begin{array}{c}\text { Proposed } \\
\text { Method }\end{array}$ \\
\hline SNR & 20.0903 & 19.2371 & 19.4068 & 21.1010 \\
\hline MSE & $1.8120 \times 10^{5}$ & $1.9048 \times 10^{5}$ & $1.9032 \times 10^{5}$ & $1.7493 \times 10^{5}$ \\
\hline RMS_SNR & 4.4822 & 4.3860 & 4.4053 & 4.5936 \\
\hline RMSE & 425.6759 & 436.4410 & 436.2544 & 418.2428 \\
\hline IFY & 0.9502 & 0.9480 & 0.9485 & 0.9526 \\
\hline
\end{tabular}

Fig. 5 is provided to visualize the changes in numerical measurements that indicate whether an image is better or not. Fig. 5 (a) compares the SNR of this proposed method with the existing methods. Greater the SNR better the image. It is nicely seen that method provides greater SNR than the existing methods. Fig. 5 (b) shows the MSE. It is better to have a smaller MSE and the chart bar showed it. This proposed method results in a smaller MSE according to the chart and the table also. In Fig. 5.c) shows that the filtering method gets a greater RMS_SNR and fortunately it is a piece of good news. Fig. 5(d) is the RMSE chart. RMSE needs to be smaller for the good quality of an image. Here, a little bit lower RMSE which is alright for the image. Finally Fig. 5 (e) contains the IFY. The values of IFY from the filters are almost the same but very little difference is still there. A better IFY than the existing methods is gotten. 


\section{CONCLUSION}

This research has been focused on effective algorithms which are used for Image denoising by using different filtering techniques. The proposed algorithm aims to detect the noise as well as remove the unwanted signal from the raw image which gives a better performance than the existing filtering technique based on SNR, MSE, RMSE, IFY, etc. In the interim, the proposed filtering technique has been proposed for denoising an image is evaluated both subjectively and objectively. Furthermore, the result in different parameters has been evaluated by using the proposed filtering technique which provides a better experimental result compare with the existing filtering techniques. In the future, developing a technique to recover images with a high percentage of noise and defects by using machine learning as well as deep learning. Then the proposed method needs to be included more parameters and evaluate the performances of the proposed technique.

\section{REFERENCES}

[1] Abdalla, A.M., Osman, M.S., AlShawabkah, H., Rumman, O., Mherat, M.: A review of nonlinear image-denoising techniques. In: 2018 Second World Conference on Smart Trends in Systems, Security and Sustainability (WorldS4). pp. 96100. IEEE (2018)

[2] Akar, S.A.: Determination of optimal parameters for bilateral filter in brain $\mathrm{mr}$ image denoising. Applied soft computing 43, 87-96 (2016)

[3] Brooks, T., Mildenhall, B., Xue, T., Chen, J., Sharlet, D., Barron, J.T.: Unprocessing images for learned raw denoising. In: Proceedings of the IEEE Conference on Computer Vision and Pattern Recognition. pp. 11036-11045 (2019)

[4] Goyal, P., Chaurasia, V.: Application of median filter in removal of random valued impulse noise from natural images. In: 2017 International conference of Electronics, Communication and Aerospace Technology (ICECA). vol. 1, pp. 125128. IEEE (2017)

[5] Islam, M.A., Talukder, M.H., Hasan, M.M.: Speckle noise reduction from ultrasound image using modified binning method and fuzzy inference system. In: 2013 2nd International Conference on Advances in Electrical Engineering (ICAEE). pp. 359-362. IEEE (2013)

[6] Khare, C., Nagwanshi, K.K.: Image restoration technique with non linear filter. International Journal of Advanced Science and Technology 39, 67-74 (2012)

[7] Kubota, A., Aizawa, K.: Reconstructing arbitrarily focused images from two differently focused images using linear filters. IEEE Transactions on Image Processing 14(11), 18481859 (2005)

[8] Lavania, K.K., Kumar, R., et al.: Image enhancement using filtering techniques. International Journal on Computer Science and Engineering 4(1), 14 (2012)

[9] Motwani, M.C., Gadiya, M.C., Motwani, R.C., Harris, F.C.: Survey of image denoising techniques. In: Proceedings of GSPX. pp. 27-30 (2004)

[10] Niharika, E., Adeeba, H., Krishna, A.S.R., Yugander, P.: Kmeans based noisy sar image segmentation using median filtering and otsu method. In: 2017 International Conference on IoT and Application (ICIOT). pp. 1-4. IEEE (2017)

[11] Pushpavalli, R., Srinivasan, E., Himavathi, S.: A new nonlinear filtering technique for image denoising. In: 2010 Inter- national Conference on Advances in Recent Technologies in Communication and Computing. pp. 1-4. IEEE (2010)

[12] Ribas, D., Ridao, P., Neira, J., Tardós, J.D.: A method for extracting lines and their uncertainty from acoustic underwater images for slam. IFAC Proceedings Volumes 40(15), 61-66 (2007)

[13] Rodrigues, I., Sanches, J., Bioucas-Dias, J.: Denoising of medical images corrupted by poisson noise. In: 2008 15th IEEE International Conference on Image Processing. pp. 1756-1759. IEEE (2008)

[14] Saravanan, M.U.M., Prabhu, T., Kumar, A.S., Jagadesh, M., Udhayamoorthi, M.: Analysis and implementation of mean, maximum and adaptive median for removing gaussian noise and salt \& pepper noise in images. European Journal of Applied Sciences 9(5), 219-223 (2017)

[15] Sarode, M.V., Deshmukh, P.R.: Reduction of speckle noise and image enhancement of images using filtering technique. International Journal of Advancements in Technology 2(1), 30-38 (2011)

[16] Sathish Kumar, P., Jerritta, S., Rajendran, V.: De-noising algorithm for snr improvement of underwater acoustic signals using cwt based on fourier transform (2018)

[17] Sathya, P., Jothi, R.A., Palanisamy, V.: Image de-noising using linear and decision based median filters (2018)

[18] Selvi, A.S., Kumar, K.P.M., Dhanasekeran, S., Maheswari, P.U., Ramesh, S., Pandi, S.S.: De-noising of images from salt and pepper noise using hybrid filter, fuzzy logic noise detector and genetic optimization algorithm (hfgoa). Multimedia Tools and Applications pp. 1-17 (2019)

[19] Senthil Selvi, A., Sukumar, R.: Removal of salt and pepper noise from images using hybrid filter (hf) and fuzzy logic noise detector (flnd). Concurrency and Computation: Practice and Experience 31(12), e4501 (2019)

[20] Suhas, S., Venugopal, C.: Mri image preprocessing and noise removal technique using linear and nonlinear filters. In: 2017 International Conference on Electrical, Electronics, Communication, Computer, and Optimization Techniques (ICEECCOT). pp. 1-4. IEEE (2017)

[21] Talukder, M.H., Rahman, M.M.: Despeckling 3d ultrasound images using linear regression. In: International Conference on Materials, Electronics \& Information Engineering, ICMEIE-2015 (2015)

[22] Talukder, M.H., Reza, M.A.I.M.M., Absar, M.A.: An optimized derivative filter for efficient edge detection of gray scale image. In: International Journal of Emerging Technologies in Computational and Applied Sciences (IJETCAS) (2013)

[23] Tania, S., Rowaida, R.: A comparative study of various image filtering techniques for removing various noisy pixels in aerial image. International Journal of Signal Processing, Image Processing and Pattern Recognition 9(3), 113-124 (2016)

[24] Vijaykumar, V., Vanathi, P., Kanagasabapathy, P.: Fast and efficient algorithm to remove gaussian noise in digital images. IAENG International Journal of Computer Science 37(1), 300-302 (2010)

[25] Xu, Y., Luo, M., Li, T., Song, G.: Ecg signal de-noising and baseline wander correction based on ceemdan and wavelet threshold. Sensors 17(12), 2754 (2017)

[26] Zhao, B., Huang, W., Wang, H.H., Liu, Z.: Image de-noising algorithm based on image reconstruction and compression 
perception. In: 2017 International Conference on Inventive Computing and Informatics (ICICI). pp. 532-535. IEEE (2017) 


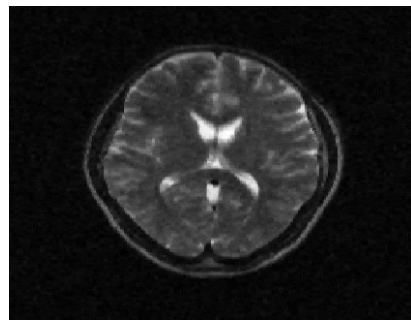

(a)

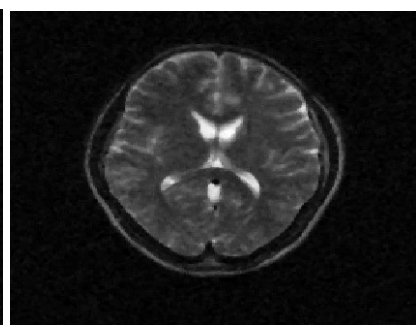

(b)

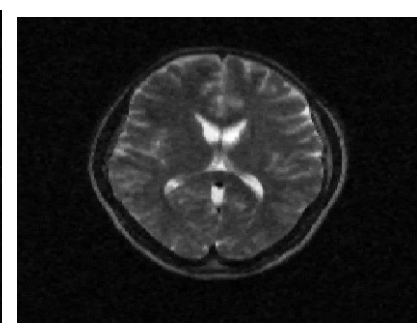

(c)

Fig. 3. (a) Original Noisy Image, (b) Filtered Image using Existing Filter, (c) Filtered Image using Proposed Technique

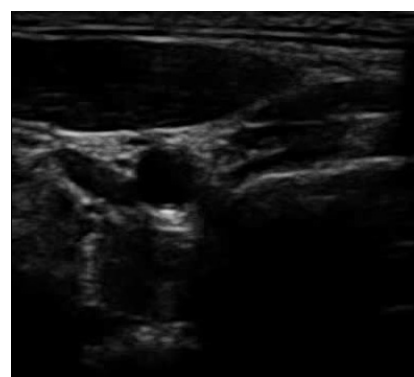

(a)

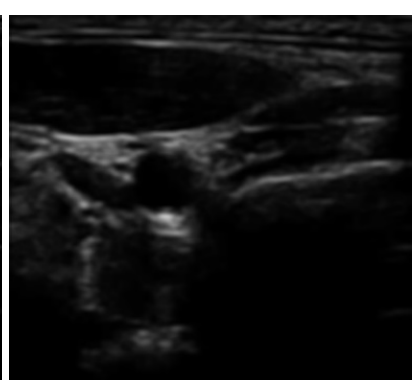

(b)

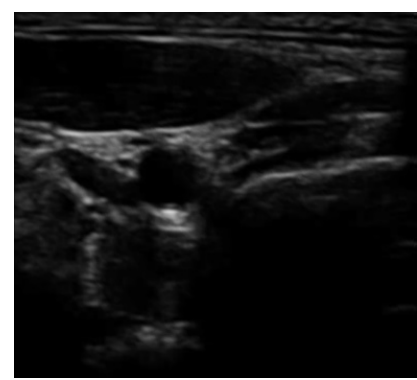

(c)

Fig. 4. (a) Raw Carotid Artery Image, (b) Filtered Image using Existing Filter, (c) Filtered Image using Proposed Technique

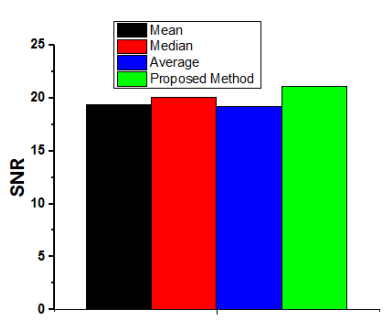

(a)

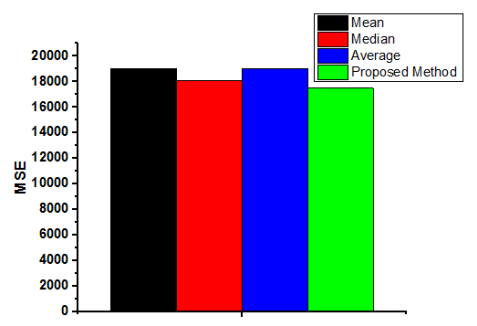

(b)

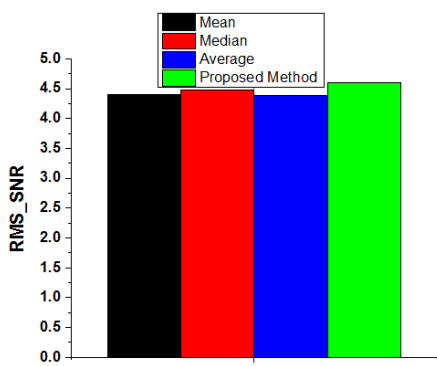

(c)

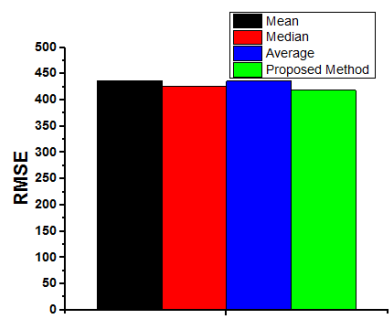

(d)

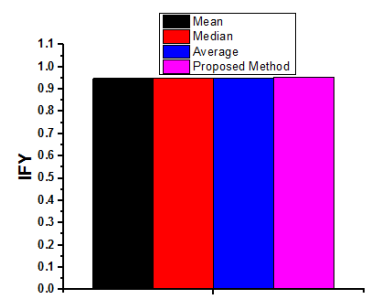

(e)

Fig. 5. Comparison of Existing Methods and Proposed Method for Brain Noisy Images (a) SNR, (b) MSE, (c) RMS_SNR, (d) RMSE, (e) IFY 